\title{
Integrator Forwarding Control Laws for Some Classes of Linearizable Feedforward Systems
}

\author{
MiroslaV KrstiC \\ Department of Mechanical and Aerospace Engineering \\ University of California at San Diego, La Jolla, CA 92093-0411
}

\begin{abstract}
In a companion paper (Krstic, "Feedforward systems linearizable by coordinate change," ACC'04) we revealed that the family of feedforward systems contains a substantial class that is linearizable by a diffeomorphic coordinate change. In this paper we present two subclasses for which explicit control formulae can be derived. Our procedures follow the general integrator forwarding algorithm of Sepulchre-JankovicKokotovic but avoid the requirements to solve (analytically) a series of nonlinear ODEs and to compute (analytically) a series of integrals with respect to time.
\end{abstract}

\section{Introduction}

In a companion paper [2] we showed that the family of feedforward systems $[6,3,1,4,5,7]$ contains a substantial class that is linearizable by a diffeomorphic coordinate change. In this paper we present two subclasses for which explicit control formulae can be derived. Our procedures follow the general integrator forwarding algorithm of Sepulchre-Jankovic-Kokotovic [5] but avoid the requirements to solve (analytically) a series of nonlinear ODEs and to compute (analytically) a series of integrals with respect to time.

The paper is organized as follows. For two important subclasses of linearizable feedforward systems, control laws are given explicitly in Sections 2 and 3. Second and third order examples of those classes of systems are presented in some detail in Section 4, shedding light on how typical, or atypical, linearizability is for feedforward systems, which is further pursued in Section 5. To comply with length restrictions, most of the proofs are omitted.

\section{Linearizable Feedforward Systems of Type I}

Consider the class of strict-feedforward systems,

$$
\begin{aligned}
\dot{x}_{1} & =x_{2}+\sum_{j=2}^{n-1} \pi_{j}\left(x_{j}\right) x_{j+1}+\pi_{n}\left(x_{n}\right) u \\
\dot{x}_{i} & =x_{i+1}, \quad i=2, \ldots, n-1 \\
\dot{x}_{n} & =u,
\end{aligned}
$$

where $\pi_{j}(0)=0$. Any system in this class is DECI [2].

Theorem 1 The diffeomorphic transformation

$$
\begin{aligned}
y_{1} & =x_{1}-\sum_{j=2}^{n} \int_{0}^{x_{j}} \pi_{j}(s) d s \\
y_{i} & =x_{i}, \quad i=2, \ldots, n
\end{aligned}
$$

converts the strict-feedforward system (1)-(3) into the chain of integrators

$$
\begin{aligned}
\dot{y}_{i} & =y_{i+1}, \quad i=1,2, \ldots, n-1 \\
\dot{y}_{n} & =u .
\end{aligned}
$$

The feedback law

$$
u=\alpha_{1}(x)=-\sum_{i=1}^{n}\left(\begin{array}{c}
n \\
i-1
\end{array}\right) y_{i}
$$

globally asymptotically stabilizes the origin of (1)-(3).

We note that in the design (4), (5), (8) we have completely circumvented the SJK procedure. It is therefore worth noting that, following the SJK procedure, one would have obtained

$$
\begin{aligned}
\alpha_{i}\left(\underline{x}_{i}\right) & =-\sum_{j=i}^{n}\left(\begin{array}{c}
n-i+1 \\
j-i
\end{array}\right) x_{j}+\delta_{i, 1} \sum_{j=2}^{n} \int_{0}^{x_{j}} \pi_{j}(s) d s(9) \\
w_{i} & =1
\end{aligned}
$$

where $\delta_{i, 1}$ denotes the Kronecker delta.

While in [2] we showed that one can avoid having to analytically solve a sequence of nonlinear ODEs, in Theorem 1 we showed that, for the feedforward subclass (1)-(3), one can also avoid having to calculate the SJK integrals (see [2]). In the next result we go even further and show that, not only does one have a closed-form formula for the control law (8) but one can even get a closed-form formula for the solutions of the system under that control law. This is not just an aesthetically pleasing result-it will allow us, in a future companion paper, to derive bounds on the control effort given explicitly in terms of the size of initial conditions. 
Theorem 2 Starting from the initial condition $x$, the solution of the feedback system (1)-(3), (4)-(8) at time $\tau$ is 1

$$
\begin{aligned}
\xi_{i}(\tau, x)= & \mathrm{e}^{-\tau}\left[\sum_{j=i}^{n}\left(\begin{array}{c}
n-i \\
j-i
\end{array}\right)(-1)^{j-i}\right. \\
& \times \sum_{k=0}^{j-1} \frac{(-\tau)^{k}}{k !} \sum_{l=j-k}^{n}\left(\begin{array}{c}
n-j+k \\
l-j+k
\end{array}\right) x_{l} \\
& +(-1)^{i} \sum_{j=i}^{n}\left(\begin{array}{c}
n-i \\
j-i
\end{array}\right) \frac{\tau^{j-1}}{(j-1) !} \\
& \left.\times\left(\sum_{m=2}^{n} \int_{0}^{x_{m}} \pi_{m}(s) d s\right)\right]
\end{aligned}
$$

for $i=2, \ldots, n$ and

$$
\begin{aligned}
\xi_{1}(\tau, x)= & \mathrm{e}^{-\tau}\left[\sum_{j=1}^{n}\left(\begin{array}{c}
n-1 \\
j-1
\end{array}\right)(-1)^{j-1}\right. \\
& \times \sum_{k=0}^{j-1} \frac{(-\tau)^{k}}{k !} \sum_{l=j-k}^{n}\left(\begin{array}{r}
n-j+k \\
l-j+k
\end{array}\right) x_{l} \\
& -\sum_{j=1}^{n}\left(\begin{array}{c}
n-1 \\
j-1
\end{array}\right) \frac{\tau^{j-1}}{(j-1) !} \\
& \left.\times\left(\sum_{m=2}^{n} \int_{0}^{x_{m}} \pi_{m}(s) d s\right)\right] \\
& +\sum_{j=2}^{n} \int_{0}^{\xi_{j}(\tau, x)} \pi_{j}(s) d s
\end{aligned}
$$

whereas the control signal is

$$
\begin{aligned}
u= & \tilde{\alpha}_{1}(\tau, x) \\
= & -\mathrm{e}^{-\tau} \sum_{i=1}^{n}\left(\begin{array}{c}
n \\
i-1
\end{array}\right)\left[\sum_{j=i}^{n}\left(\begin{array}{c}
n-i \\
j-i
\end{array}\right)(-1)^{j-i}\right. \\
& \times \sum_{k=0}^{j-1} \frac{(-\tau)^{k}}{k !} \sum_{l=j-k}^{n}\left(\begin{array}{c}
n-j+k \\
l-j+k
\end{array}\right) x_{l} \\
& +(-1)^{i} \sum_{j=i}^{n}\left(\begin{array}{c}
n-i \\
j-i
\end{array}\right) \frac{\tau^{j-1}}{(j-1) !} \\
& \times\left(\sum_{m=2}^{n} \int_{0}^{x_{m}} \pi_{m}(s) d s\right)
\end{aligned}
$$

\footnotetext{
${ }^{1}$ We emphasize that $x$, which denotes the initial condition, is constant. We also point out that, relative to the notation in [2], $\xi(\tau, x)$ and $\zeta(\tau, z)$ should be understood, respectively, as $\xi^{[1]}(\tau, x)$ and $\zeta^{[1]}(\tau, z)$.
}

\section{Linearizable Feedforward Systems of Type II}

Consider the class of the strict-feedforward systems

$$
\begin{aligned}
\dot{x}_{i} & =x_{i+1}+\phi_{i}\left(\underline{x}_{i+1}\right) u, \quad i=1, \ldots, n-1( \\
\dot{x}_{n} & =u
\end{aligned}
$$

where $\phi_{i}(0)=0$. In this section we construct control laws for a linearizable subclass of (14), (15).

To characterize the linearizable subclass, let us consider the functions $\phi_{n-1}\left(x_{n}\right)$ and $\phi_{i}\left(0, \ldots, 0, x_{n}\right), i=$ $1, \ldots, n-2$, as given and introduce the following sequence of functions:

$$
\begin{aligned}
\mu_{n}\left(x_{n}\right)= & \frac{\int_{0}^{x_{n}} \phi_{n-1}(s) d s}{x_{n}} \\
\mu_{i}\left(x_{n}\right)= & \frac{1}{x_{n}} \int_{0}^{x_{n}}\left[\phi_{i-1}(0, \ldots, 0, s)\right. \\
& \left.-\sum_{j=i+1}^{n} \mu_{j}(s) \phi_{i+n-j}(0, \ldots, 0, s)\right]
\end{aligned}
$$

for $i=n-1, n-2, \ldots, 2$, and

$\gamma_{1}\left(x_{n}\right)=\mu_{n}^{\prime}\left(x_{n}\right)$

$\gamma_{k}\left(x_{n}\right)=\sum_{l=1}^{k-1} \gamma_{l}\left(x_{n}\right) \mu_{l+n+1-k}\left(x_{n}\right)+\frac{d \mu_{n+1-k}\left(x_{n}\right)}{d x_{n}}$

for $k=2, \ldots, n-2$.

\section{Theorem 3 If}

$$
\phi_{i}\left(\underline{x}_{i+1}\right)=\sum_{j=i+1}^{n-1} \gamma_{j-i}\left(x_{n}\right) x_{j}+\phi_{i}\left(0, \ldots, 0, x_{n}\right)
$$

$\forall x, i=1, \ldots, n-2$, then the diffeomorphic transformation

$$
\begin{aligned}
y_{i} & =x_{i}-\sum_{j=i+1}^{n} \mu_{i+1+n-j}\left(x_{n}\right) x_{j}, \quad i=1, \ldots, n-1 \\
y_{n} & =x_{n}
\end{aligned}
$$

converts the strict-feedforward system (14)-(15) into the chain of integrators (6)-(7). The feedback law

$$
u=\alpha_{1}(x)=-\sum_{i=1}^{n}\left(\begin{array}{c}
n \\
i-1
\end{array}\right) y_{i}
$$

globally asymptotically stabilizes the origin of (14)(15). 
As in Section 2, we point out that, following the SJK procedure, one would have obtained

$$
\begin{aligned}
\alpha_{i}\left(\underline{x}_{i}\right)= & -x_{i}-\sum_{m=i+1}^{n} x_{m}\left[\left(\begin{array}{c}
n-i+1 \\
m-i
\end{array}\right)\right. \\
& \left.-\sum_{j=i}^{m}\left(\begin{array}{c}
n-i+1 \\
j-i
\end{array}\right) \mu_{j+1+n-m}\left(x_{n}\right)\right](24) \\
w_{i}= & 1 .
\end{aligned}
$$

Example 1 To illustrate the above concepts (and notation), let us consider a fourth order example of a Type II feedforward system:

$$
\begin{aligned}
\dot{x}_{1} & =x_{2}+\left(\frac{x_{2}}{2}-\frac{x_{3} x_{4}}{12}\right) u \\
\dot{x}_{2} & =x_{3}+\frac{x_{3}}{2} u \\
\dot{x}_{3} & =x_{4}+x_{4} u \\
\dot{x}_{4} & =u .
\end{aligned}
$$

The control law

$$
\begin{aligned}
u & =-y_{1}-4 y_{2}-6 y_{3}-4 y_{4} \\
& =-z_{1}-z_{2}-z_{3}-z_{4},
\end{aligned}
$$

where

$$
\begin{aligned}
& y_{1}=x_{1}-\frac{x_{4} x_{2}}{2}+\frac{x_{4}^{2} x_{3}}{6}-\frac{x_{4}^{4}}{24} \\
& y_{2}=x_{2}-\frac{x_{4} x_{3}}{2}+\frac{x_{4}^{3}}{6} \\
& y_{3}=x_{3}-\frac{x_{4}^{2}}{2} \\
& y_{4}=x_{4},
\end{aligned}
$$

which is obtained with

$$
\begin{aligned}
& \mu_{2}=\frac{x_{4}^{3}}{24} \\
& \mu_{3}=-\frac{x_{4}^{2}}{6} \\
& \mu_{4}=\frac{x_{4}}{2},
\end{aligned}
$$

and $z_{i}=x_{i}-\beta_{i+1}$ with

$$
\begin{aligned}
\beta_{4}= & \left(\frac{x_{4}}{2}-1\right) x_{4} \\
\beta_{3}= & \left(\frac{x_{4}}{2}-2\right) x_{3}-x_{4}+x_{4}^{2}-\frac{x_{4}^{3}}{6} \\
\beta_{2}= & \left(\frac{x_{4}}{2}-3\right) x_{2}+\left(-3+\frac{3}{2} x_{4}-\frac{x_{4}^{2}}{6}\right) x_{3} \\
& -x_{4}-\frac{3}{2} x_{4}^{2}+\frac{1}{2} x_{4}^{3}-\frac{1}{24} x_{4}^{4}
\end{aligned}
$$

achieves

$$
\dot{z}=\left[\begin{array}{cccc}
-1 & 0 & 0 & 0 \\
-1 & -1 & 0 & 0 \\
-1 & -1 & -1 & 0 \\
-1 & -1 & -1 & -1
\end{array}\right] z
$$

and $(s+1)^{3} y_{1}(s)=0$.

For using the results of this section for control designs beyond the Type II class of systems, we need the inverse of the coordinate transformation (21). The explicit form of the inverse transformation is given in the following theorem.

Lemma 1 Consider the series of functions

$$
\begin{aligned}
\lambda_{n}\left(x_{n}\right)= & \mu_{n}\left(x_{n}\right) \\
\lambda_{i}\left(x_{n}\right)= & \frac{1}{x_{n}} \int_{0}^{x_{n}}\left(s \sum_{l=i+1}^{n} \gamma_{l-i}(s) \lambda_{l}(s)\right. \\
& \left.+\phi_{i-1}(0, \ldots, 0, s)\right) d s
\end{aligned}
$$

for $i=n-1, \ldots, 2$. The inverse of the diffeomorphic transformation (21) is

$$
\begin{aligned}
x_{i} & =y_{i}+\sum_{j=i+1}^{n} \lambda_{i+1+n-j}\left(y_{n}\right) y_{j}, \quad i=1, \ldots, n-1 \\
x_{n} & =y_{n} .
\end{aligned}
$$

As in Theorem 2, in the next result we give a closedform formula for the solutions of the feedback system from Theorem 3 .

Theorem 4 Starting from the initial condition $x$, the solution of the feedback system (14)-(20), (23) at time $\tau$ 
is

$$
\begin{aligned}
\xi_{i}(\tau, x)= & \mathrm{e}^{-\tau}\left[\sum_{j=i}^{n}\left(\begin{array}{c}
n-i \\
j-i
\end{array}\right)(-1)^{j-i}\right. \\
& \times \sum_{k=0}^{j-1} \frac{(-\tau)^{k}}{k !} \sum_{l=j-k}^{n}\left(\begin{array}{c}
n-j+k \\
l-j+k
\end{array}\right) \\
& \times\left(x_{l}-\sum_{m=l+1}^{n} \mu_{l+1+n-m}\left(x_{n}\right) x_{m}\right) \\
& +\sum_{p=i+1}^{n} \lambda_{i+1+n-p}\left(\mathrm{e}^{-\tau} \sum_{k=0}^{n-1} \frac{(-\tau)^{k}}{k !}\right. \\
& \times \sum_{l=n-k}^{n}\left(\begin{array}{c}
l-n+k \\
k
\end{array}\right) \\
& \left.\times\left(x_{l}-\sum_{m=l+1}^{n} \mu_{l+1+n-m}\left(x_{n}\right) x_{m}\right)\right) \\
& \times \sum_{j=p}^{n}\left(\begin{array}{c}
n-p \\
j-p
\end{array}\right)(-1)^{j-p} \\
& \times \sum_{k=0}^{j-1} \frac{(-\tau)^{k}}{k !} \sum_{l=j-k}^{n}\left(\begin{array}{c}
n-j+k \\
l-j+k
\end{array}\right) \\
& \times\left(x_{l}-\sum_{m=l+1}^{n} \mu_{l+1+n-m}\left(x_{n}\right) x_{m}\right)
\end{aligned}
$$

where $i=1, \ldots, n$, and the control signal is

$$
\begin{aligned}
u= & \tilde{\alpha}_{1}(\tau, x) \\
= & -\mathrm{e}^{-\tau} \sum_{i=1}^{n}\left(\begin{array}{c}
n \\
i-1
\end{array}\right) \sum_{j=i}^{n}\left(\begin{array}{c}
n-i \\
j-i
\end{array}\right)(-1)^{j-i} \\
& \times \sum_{k=0}^{j-1} \frac{(-\tau)^{k}}{k !} \sum_{l=j-k}^{n}\left(\begin{array}{c}
n-j+k \\
l-j+k
\end{array}\right) \\
& \times\left(x_{l}-\sum_{m=l+1}^{n} \mu_{l+1+n-m}\left(x_{n}\right) x_{m}\right) .
\end{aligned}
$$

\section{Type I and II Systems in Dimensions Two and Three}

We start by pointing out that in dimension two all strictfeedforward systems are simultaneously of Types I and II. This implies that all second order strict-feedforward systems are linearizable.

Theorem 5 Consider the system

$$
\begin{aligned}
& \dot{x}_{1}=x_{2}+\phi_{1}\left(x_{2}\right) u \\
& \dot{x}_{2}=u
\end{aligned}
$$

where $\phi_{1}(0)$ is continuous and $\phi_{1}(0)=0$. The control law

$$
u=-x_{1}-2 x_{2}+\int_{0}^{x_{2}} \phi_{1}(s) d s
$$

ensures global asymptotic stability of the origin.

Example 2 Let us now consider an example with $\phi_{1}\left(x_{2}\right)=-x_{2}^{2}$. This example was worked out in [4]. In this case the formula (51) gives $^{2}$

$$
u=-x_{1}-2 x_{2}-\frac{x_{2}^{3}}{3}
$$

One should recognize that the " $-x_{1}-2 x_{2}$ " portion of the control law (52) is responsible for exponential stabilization of the linearized system. To see that this linear controller is not sufficient for global stabilization, we plug it back into the plant and obtain a closed loop system, written in the form of a second order equation, as

$$
\ddot{x}_{2}+\left(2-x_{2}^{2}\right) \dot{x}_{2}+x_{2}=0 \text {. }
$$

This is a Van der Pol equation with an unstable limit cycle, which exhibits a finite escape instability. Hence, the nonlinear term “- $-\frac{x_{2}^{3}}{3}$," designed to accommodate the input nonlinearity $\phi_{1}\left(x_{2}\right)=-x_{2}^{2}$, is crucial for global stabilization.

The possibilities, as well as the limits, of Type I/II linearizability for strict-feedforward systems are best understood in dimension three. The following class of systems, which represents a union of all Type I and Type II feedforward systems in dimension three, is linearized in the next theorem.

Theorem 6 Consider the class of systems

$$
\begin{aligned}
\dot{x}_{1}= & x_{2}+\pi_{2}\left(x_{2}\right) x_{3} \\
& +\left(\frac{x_{3} \phi_{2}\left(x_{3}\right)-\int_{0}^{x_{3}} \phi_{2}(s) d s}{x_{3}^{2}} x_{2}+\pi_{3}\left(x_{3}\right)\right) u \\
\dot{x}_{2}= & x_{3}+\phi_{2}\left(x_{3}\right) u \\
\dot{x}_{3}= & u
\end{aligned}
$$

where $\pi_{2}(\cdot), \pi_{3}(\cdot) \in C^{0}$ and $\phi_{2}(\cdot) \in C^{1}$ are vanishing at the origin and

$$
\pi_{2}\left(x_{2}\right) \phi_{2}\left(x_{3}\right) \equiv 0 .
$$

\footnotetext{
${ }^{2} \mathrm{~A}$ reader checking back the details in [4] will notice that this control law differs from (6.2.12) in [4]. This is due to an extra " $x_{3}^{2}$ ", term that has crept into the calculations in [4], in equation (6.2.7). If it wasn't for this little mistake in algebra, feedback linearizability would have undoubtedly been apparent to the authors of [4].
} 
Then the control law

$$
u=-y_{1}-3 y_{2}-3 y_{3}
$$

where

$$
\begin{aligned}
y_{1}= & x_{1}-\int_{0}^{x_{2}} \pi_{2}(s) d s-\mu_{3}\left(x_{3}\right) x_{2}-\int_{0}^{x_{3}} \pi_{3}(s) d s \\
& +\frac{1}{2} x_{3}\left(\mu_{3}\left(x_{3}\right)\right)^{2}+\frac{1}{2} \int_{0}^{x_{3}}\left(\mu_{3}(s)\right)^{2} d s \\
y_{2}= & x_{2}-\int_{0}^{x_{3}} \phi_{2}(s) d s \\
y_{3}= & x_{3}
\end{aligned}
$$

and

$$
\mu_{3}\left(x_{3}\right)=\frac{\int_{0}^{x_{3}} \phi_{2}(s) d s}{x_{3}},
$$

achieves global asymptotic stability of the origin.

Proof. On can verify that $\dddot{y}_{1}+3 \ddot{y}_{1}+3 \dot{y}_{1}+y_{1}=0$

A Type II example of a system from this class is

$$
\begin{aligned}
& \dot{x}_{1}=x_{2}+\left(\frac{\mathbf{1}}{\mathbf{2}} x_{2}+x_{3} \sin x_{3}\right) u \\
& \dot{x}_{2}=x_{3}+x_{3} u \\
& \dot{x}_{3}=u,
\end{aligned}
$$

which is stabilized (and feedback linearized) using

$$
\begin{aligned}
u= & -x_{1}-3 x_{2}-3 x_{3}+\frac{x_{2} x_{3}}{2}+\frac{3}{2} x_{3}^{2} \\
& -\frac{1}{6} x_{3}^{3}+x_{3} \sin x_{3}+\cos x_{3}-1 .
\end{aligned}
$$

We point out that the key restriction in this example is the boldfaced $1 / 2$. If this value were anything else (say, 1 , or 0 ), this system would not be linearizable.

The focus on third order systems is partly motivated by the fact that the celebrated "benchmark problem"

$$
\begin{aligned}
& \dot{x}_{1}=x_{2}+x_{3}^{2} \\
& \dot{x}_{2}=x_{3} \\
& \dot{x}_{3}=u,
\end{aligned}
$$

first solved by Teel [6] using his method of nested saturations, is of third order. The system (67)-(69) is not feedback linearizable. However, the following similar (at least visually) systems, are linearizable. The system

$$
\begin{aligned}
\dot{x}_{1} & =x_{2}+x_{3}^{2} u \\
\dot{x}_{2} & =x_{3} \\
\dot{x}_{3} & =u
\end{aligned}
$$

is linearizable, as it is of both Type I and Type II. The system

$$
\begin{aligned}
& \dot{x}_{1}=x_{2}+x_{2}^{2} x_{3} \\
& \dot{x}_{2}=x_{3} \\
& \dot{x}_{3}=u
\end{aligned}
$$

is of Type I, and therefore linearizable. Other such systems exist, outside of Types I or II, that are linearizable. For example,

$$
\begin{aligned}
& \dot{x}_{1}=x_{2}+x_{3}^{2}+x_{2} u \\
& \dot{x}_{2}=x_{3} \\
& \dot{x}_{3}=u
\end{aligned}
$$

(which is temptingly close in appearance to Type I but is not in that class), is linearizable using the coordinate change

$$
\begin{aligned}
& y_{1}=x_{1}-x_{2} x_{3} \\
& y_{2}=x_{2} \\
& y_{3}=x_{3} .
\end{aligned}
$$

The above examples all had the last two equations actually linear. The neither-Type-I-nor-II feedforward system

$$
\begin{aligned}
\dot{x}_{1} & =x_{2}+x_{2}^{2} x_{3}+\frac{x_{3}^{2}}{3}-x_{2}^{2} x_{3}^{2} u \\
\dot{x}_{2} & =x_{3}-x_{3}^{2} u \\
\dot{x}_{3} & =u,
\end{aligned}
$$

which includes nonlinearities in both of the first two equations, is linearizable using the coordinate change

$$
\begin{aligned}
& y_{1}=x_{1}-\frac{x_{2}^{3}}{3} \\
& y_{2}=x_{2}+\frac{x_{3}^{3}}{3} \\
& y_{3}=x_{3} .
\end{aligned}
$$

Clearly, since the systems (76)-(78) and (82)-(84) are neither of Type I nor II, the coordinate changes (79)(81) and (85)-(87) cannot be obtained from the explicit formulae in Sections 2 and 3. However, they can be obtained following the simplified SJK procedure in [2], which, we remind the reader, avoids the requirement to solve a series of nonlinear ODEs.

\section{5 (In Lieu of) Conclusions: More on Type I and II Systems}

How generic, or nongeneric, is linearizability within the class of strict-feedforward systems? It is hard to quantitatively state what "percentage" of feedforward system 
are linearizable, or, how close (in some metric) a feedforward system is to a linearizable feedforward system. However, it is clear from the results of this paper that one should not expect the majority of feedforward systems to be linearizable.

The Type II class is particularly interesting because of its structural peculiarity (recall (16)-(20)). Based on the third order case where any linearizable system with $\psi_{1}\left(x_{2}, x_{3}\right) \equiv 0$ is of Type II, one might be tempted to conjecture that all linearizable systems (of any order) with $\psi_{i}\left(\underline{x}_{i+1}\right) \equiv 0, i=1, \ldots, n-2$ are of Type II. ${ }^{3} \mathrm{~A}$ fourth order counter-example to this conjecture is

$$
\begin{aligned}
& \dot{x}_{1}=x_{2}+\left(2 x_{2}^{2} x_{4}-x_{2} x_{3}^{2}\right) u \\
& \dot{x}_{2}=x_{3} \\
& \dot{x}_{3}=x_{4} \\
& \dot{x}_{4}=u,
\end{aligned}
$$

which is linearizable via coordinate change

$$
\begin{gathered}
y_{1}=x_{1}-\left(x_{2} x_{4}-\frac{x_{3}^{2}}{2}\right)^{2} \\
y_{2}=x_{2}, \quad y_{3}=x_{3}, \quad y_{4}=x_{4}
\end{gathered}
$$

but is not of Type II.

It is not clear at this point what the avenues for possible generalization of the results of this paper might be. The most immediate idea would be to start by exploring the possibilities for combining the systems of Type I and Type II. Theorem 6 does this, at least notationally, for systems of order three. The condition (57) shows actually that these two classes do not mix well, i.e., that Theorem 6 is a concise statement of two results, not a statement for a mixed Type I/II class. However, while mixing is impossible in order three, it is not impossible in higher orders. For example, the fourth order system

$$
\begin{aligned}
& \dot{x}_{1}=x_{2}+\frac{x_{3} a\left(x_{3}\right)-\int_{0}^{x_{3}} a(s) d s}{x_{3}^{2}} x_{2} x_{4}+b\left(x_{4}\right) u \\
& \dot{x}_{2}=x_{3}+a\left(x_{3}\right) x_{4} \\
& \dot{x}_{3}=x_{4} \\
& \dot{x}_{4}=u,
\end{aligned}
$$

where $a(\cdot)$ and $b(\cdot)$ are any nonlinearities vanishing at zero ( $a$ also must be $C^{1}$ ), is a system that mixes the features of Types I and II and is linearizable via

$$
\begin{aligned}
y_{1}= & x_{1}-\mu\left(x_{3}\right) x_{2}+\frac{1}{2} x_{3}\left(\mu\left(x_{3}\right)\right)^{2} \\
& +\frac{1}{2} \int_{0}^{x_{3}}(\mu(s))^{2} d s-\int_{0}^{x_{4}} b(s) d s \\
y_{2}= & x_{2}-\int_{0}^{x_{3}} a(s) d s \\
y_{3}= & x_{3} \\
y_{4}= & x_{4},
\end{aligned}
$$

where

$$
\mu\left(x_{3}\right)=\frac{\int_{0}^{x_{3}} a(s) d s}{x_{3}} .
$$

\section{References}

[1] M. Jankovic, R. Sepulchre, and P. V. Kokotovic, "Constructive Lyapunov stabilization of nonlinear cascade systems," IEEE Transactions on Automatic Control, vol. 41, pp. 1723-1735, 1996.

[2] M. Krstic, "Feedforward systems linearizable by coordinate change," 2004 American Control Conference.

[3] F. Mazenc and L. Praly, "Adding integrations, saturated controls, and stabilization of feedforward systems," IEEE Transactions on Automatic Control, vol. 41, pp. 1559-1578, 1996.

[4] R. Sepulchre, M. Jankovic, and P. Kokotovic, Constructive Nonlinear Control, Springer, 1997.

[5] R. Sepulchre, M. Jankovic, and P. Kokotovic, "Integrator forwarding: A new recursive nonlinear robust design," Automatica, pp. 979-984, vol. 33, 1997.

[6] A. R. Teel, Feedback Stabilization: Nonlinear Solutions to Inherently Nonlinear Problems, PhD dissertation, University of California, Berkeley, 1992.

[7] A. R. Teel, "A nonlinear small gain theorem for the analysis of control systems with saturation," IEEE Transactions on Automatic Control, vol. 41, pp. 12561270, 1996.

\footnotetext{
${ }^{3}$ We remind the reader that $\psi_{n-1}\left(x_{n}\right) \equiv 0[2]$.
} 